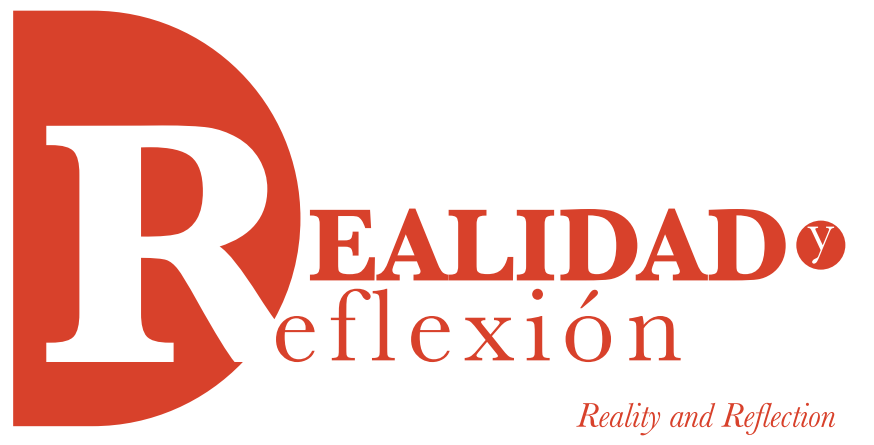

ISSN 1992-6510

e-ISSN 2520-92990

Año 17, N 45, San Salvador, El Salvador, Centroamérica. Revista Semestral Enero-Junio 2017

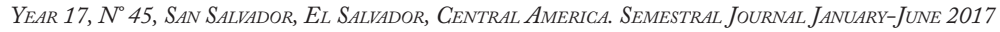

\title{
Evaluación de impacto del Programa Empresa Centro en E1 Salvador
}

\author{
Evaluation about the impact of the Center Company \\ program in El Salvador
}

Ricardo Olmos Guevara

Economista de la Universidad de Québec (Montreal) y de la Universidad Centroamericana José Simeón Cañas. Maestría en Finanzas de la Universidad de El Salvador. En proceso de tesis doctoral en Economía de la Universidad del Zulia, Venezuela

rholmos@hotmail.com

Fecha de recepción: 10 de enero de 2017

Fecha de aceptación: 12 de mayo de 2017

\section{RESUMEN}

El Programa Empresa Centro constituye una de las oportunidades de formación profesional en E1 Salvador, que apoyan tanto a la población vulnerable y con deseos de formación y a las empresas privadas interesadas en mano de obra especializada. Los resultados indican que los cursos de actualización y formación profesional son claves para incidir en el bienestar del participante y en el de su familia, dado que se eleva la condición de empleabilidad y mejora sustantivamente los ingresos. El autor aplica las herramientas econométricas para determinar los impactos en la formación profesional en materia de empleo y el ingreso utilizando para tal fin el enfoque econométrico de la doble diferencia.

\section{ABSTRACT}

The program "Empresa Centro" comprise one of the opportunities of vocational training in El Salvador that they support so much the vulnerable population and with desires of formation and to the private company been interested in specializing manpower. The results indicate that the courses of update and vocational training are key to affect the well-being of the participant and of his family provided that the condition rises of employability and improves substantively the income. The author applies the tools econometrics to determine the impacts in the vocational training as for employment and the revenue using for such an end the approach econometrical of the double difference. 


\section{Introducción}

Esta investigación es la síntesis de un estudio realizado para evaluar los impactos de la oferta formativa que se extiende a través del Instituto Salvadoreño de Formación Profesional (INSAFORP), del Programa Empresa Centro, PEC 2010-2011, a la población desempleada y subempleada, así como a otros grupos poblacionales que demandan capacitación técnica.

La ejecución de esta investigación corresponde a una consultoría realizada por el autor, y los resultados revelan que potenciar las condiciones básicas de formación permite elevar la empleabilidad de los participantes, observando la inserción productiva efectiva en el corto plazo.

Este estudio lleva como propósito central el hecho de que las autoridades de INSAFORP, los organismos cooperantes y la ciudadanía en general conozcan los impactos en materia laboral, ingresos y demás aspectos socioeconómicos que se vinculan con el bienestar de la población participante.

El Sistema Nacional de Formación Profesional de INSAFORP promueve y articula la demanda con la oferta de los programas que se impulsan, para que la evaluación de los mismos se monitoree de manera constante.

Profundizar y elevar el capital humano de los participantes en los programas que se impulsan por INSAFORP tienden en el corto plazo a mejorar las competencias técnicas y, en definitiva, a mejorar la productividad del trabajo, con modalidades diferentes de formación y con un menú de carreras que satisfagan la demanda y que esté orientada a lo que el país y su aparato productivo demanda.

$\mathrm{Y}$ en efecto, los aspectos que impactan directamente la empleabilidad están referidos a las oportunidades de formación profesional en cada una de las carreras de formación que forman parte de la oferta del PEC, el nivel socioeconómico del cual provienen, el género, nivel educativo, situación de la condición familiar y la zona de residencia son algunos de los elementos que, como otros, pesan por el proceso de inserción laboral. Esos aspectos están a la base de las condiciones elementales de un determinando nivel de impacto. ${ }^{1}$

En América Latina las instituciones que están vinculadas con la formación para aquellos que se encuentran desempleados o que no obstante tengan un empleo y desean actualizarse, constituye parte del paradigma que se encuentra a la base de la formación laboral y que establece para determinados sectores sociales como el instrumento más apropiado para reducir las probabilidades de caer y mantenerse en condiciones de pobreza, en espacios intergeneracionales amplios.

A lo largo de este estudio se definen los impactos del PEC y se presentan los resultados que se encuentran a la base, resultado de un extenso diálogo con los participantes. Se observa que la población que demanda los servicios de formación profesional participa e ingresa al Programa sin restricción de ninguna naturaleza.

También, es de mencionar que la cobertura de

1 Cfr. Gallart, María Antonia, La formación para el trabajo y los jóvenes en América Latina, Santiago de Chile 2003, 40p. 
la medición del impacto es global; es decir, para los 1,170 participantes en las 20 carreras a escala nacional, y es medida a través de la muestra seleccionada de 122 participantes. La inserción productiva pasa desde aquellas oportunidades en los nichos ocupacionales del mercado, que son precisamente aquellos en los que el aprendizaje en el trabajo y el valor de la experiencia es menor, así como las empresas que forman parte de las oportunidades de las que goza cada uno de los participantes del Programa. ${ }^{2} \mathrm{Y}$ de esa manera se transita hacia las ocupaciones a tiempo completo con las mayores prestaciones sociales, y que la Organización Internacional del Trabajo, OIT, lo denomina empleo decente.

La tesis central que se presenta consiste en que los jóvenes participantes con mayor formación profesional logran impactos positivos mejorando la calidad y el bienestar de ellos y de su núcleo familiar, incidiendo en la reducción sustantiva de la pobreza en E1 Salvador.

\section{Aplicación del modelo de diferencia en diferencia}

\subsection{Modelo de diferencia en diferencia del Programa Empresa Centro: el caso del empleo}

El método de diferencia en diferencia permite conocer los aspectos de impacto del Programa frente al grupo de comparación. Este método consiste, como indica su nombre, en aplicar una doble diferencia comparando los cambios a lo largo del tiempo en la variable de interés entre una población inscrita en un programa (grupo

2 INSAFORP, Evaluación de impacto: incidencia de la capacitación para el trabajo en la incorporación al empleo, de la población egresada del programa habilitación para el trabajo, años 2001 y 2002", Bases de datos. de tratamiento) y una población no inscrita (el grupo de comparación). ${ }^{3}$ (Ver Tabla n. $\left.{ }^{\circ} 1\right)$

En el caso de este estudio los impactos son más que evidentes pues la primera diferencia indica que efectivamente los participantes han aprovechado los cursos de formación profesional, ya que una vez obtuvieron la formación, su actitud hacia el empleo es más que evidente, habiendo mejorado en un $195.4 \%$ su condición laboral luego de los cursos de formación. En otras palabras, las capacidades de formación han duplicado las oportunidades de empleo en los participantes, una vez se han entregado esas oportunidades de formación por el INSAFORP. A nivel de impacto, aplicando el método de la doble diferencia, en términos absolutos el nivel de 437 puntos indica que las capacidades de impacto en materia de empleo son 4.3 veces superiores a las capacidades iniciales con las cuales se presentaron los participantes, previo a los cursos de formación.

Ello ha generado inserción productiva de los participantes y, por supuesto, mayor incidencia en su bienestar personal y de la familia. (Ver Figura n. ${ }^{\circ} 1$ )

En síntesis, el resultado de la diferencia entre el grupo de tratamiento y el grupo de comparación en relación con el empleo indica que la velocidad de incremento del empleo en el grupo de tratamiento es superior en 437 puntos versus el grupo de comparación.

Los participantes del PEC incrementaron su inserción productiva en 195.4\%; mientras que el grupo de comparación lo hizo con un 14.5\%.

\footnotetext{
3 DIGESTYC. (2012). Encuesta de Hogares de Propósitos Múltiples, p.19.
} 
Tabla n. ${ }^{\circ} 1$

El Salvador. Impacto del PEC según el método de la diferencia en diferencias en materia de empleo entre los grupos de tratamiento y grupo de comparación

\begin{tabular}{|l|c|c|c|}
\hline & Después & Antes & \\
\hline Grupo de tratamiento & $\mathrm{B}$ & $\mathrm{A}$ & $\mathrm{B}-\mathrm{A}$ \\
\hline Grupo de comparación & $\mathrm{D}$ & $\mathrm{C}$ & $\mathrm{D}-\mathrm{C}$ \\
\hline Diferencia & $\mathrm{B}-\mathrm{D}$ & $\mathrm{A}-\mathrm{C}$ & $\mathrm{DD}=(\mathrm{B}-\mathrm{A})-(\mathrm{D}-\mathrm{C})$ \\
\hline \multicolumn{4}{|c|}{} \\
\hline \multicolumn{4}{|c|}{ Antes } \\
\hline Grupo de tratamiento & Después & 263.00 & 514.00 \\
\hline Grupo de comparación & 777.00 & 531 & 77.0 \\
\hline Diferencia & 6.8 & -268.00 & $\mathbf{4 3 7 . 0 0}$ \\
\hline
\end{tabular}

Fuente: Elaboración propia.

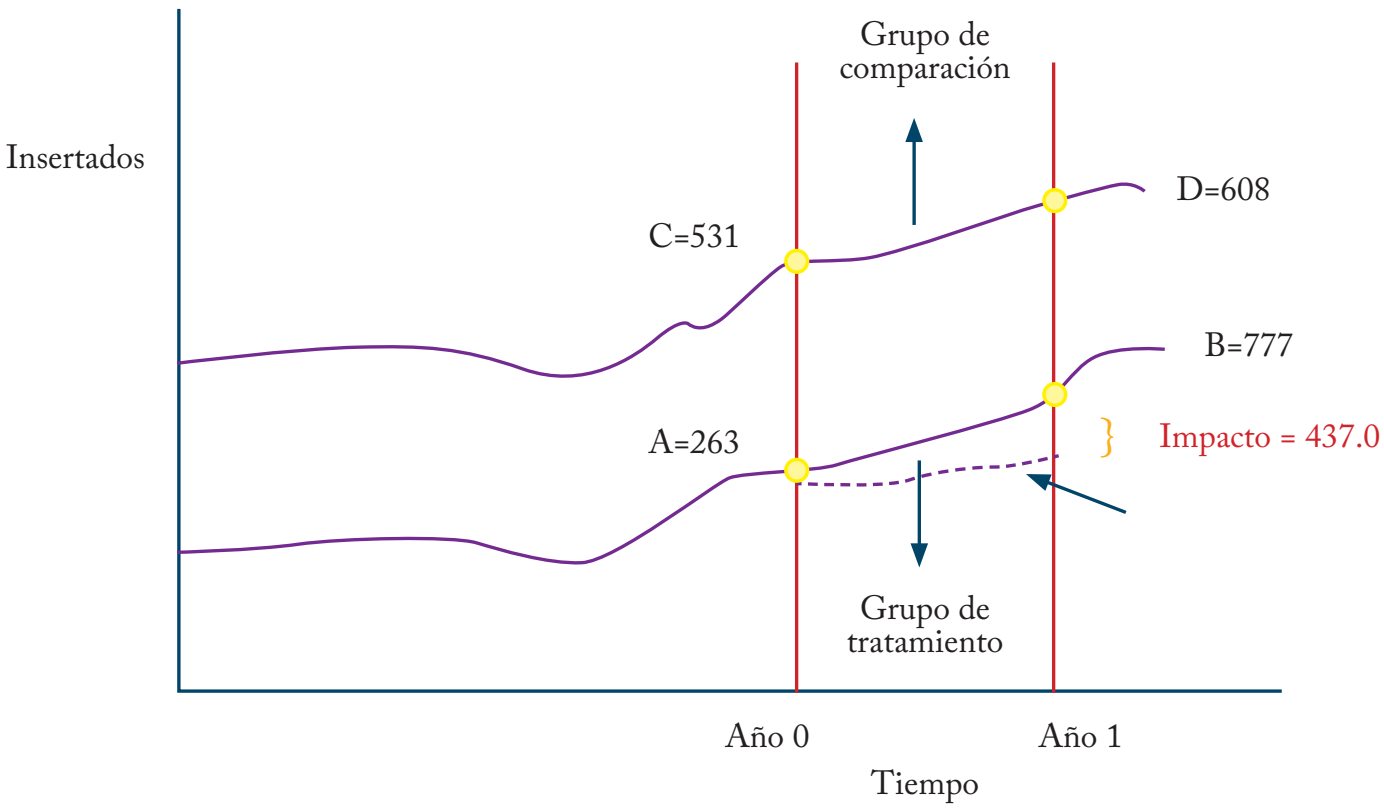

Figura n. ${ }^{\circ}$ 1. El Salvador. Evaluación de impacto del empleo del PEC. Fuente: Elaboración propia. 
2.2. El modelo de diferencia en diferencia del Programa Empresa Centro: el caso de los ingresos

El resultado de la diferencia entre el grupo de tratamiento y el grupo de comparación indica que la velocidad de incremento de los ingresos en el grupo de tratamiento es superior en 160.4 puntos versus el grupo de comparación. (Ver Tabla n. ${ }^{\circ}$ ).

La variación de los ingresos del grupo de tratamiento es de 97.9\%, que corresponde a la primera diferencia en 176.7 puntos, lo que indica que los participantes mejoraron sus ingresos una vez recibieron los cursos de formación y ganaron espacios de empleabilidad mayor para ser sujetos de opciones y oportunidades de empleo,y de ello se han derivado condiciones favorables para mejorar sus ingresos versus el grupo de comparación.

En el caso del grupo de comparación se han experimentado variaciones solo del 4.5\%, que en términos absolutos significa una mejoría de 16.3 puntos.

Finalmente, se presenta la evidencia del impacto del Programa Empresa Centro en los ingresos de los participantes, bajo el funcionamiento de este programa, mejoraron su condición de vida 1.6 veces de los que recibieron los cursos de formación frente al grupo de comparación. (Ver Figura n. 2).

En síntesis, el estudio presenta resultados favorables para aquellos participantes en el PEC, pues en los participantes de cada uno de los aspectos considerados: educación, salud, empleabilidad, ingresos, etc., se nota que en efecto hay un impacto sumamente fuerte y por encima de aquel que no se somete a este tipo de iniciativas de formación.

El método de diferencias en diferencias computa la estimación del impacto de la siguiente manera:

a. Se calcula la diferencia del resultado $(Y)$ entre las situaciones antes y después para el grupo de tratamiento (B-A).

a. Se calcula la diferencia del resultado $(Y)$ entre las situaciones antes y después para el grupo de comparación (D-C).

a. A continuación se calcula la diferencia entre la diferencia en los resultados del grupo de tratamiento $(\mathrm{B}-\mathrm{A})$ y la diferencia del grupo de comparación $(\mathrm{D}-\mathrm{C})$, o $\mathrm{DD}=(\mathrm{B}-\mathrm{A})-$ (D-C). Esta "diferencias en diferencias es la estimación de impacto". ${ }^{4}$

3. Aplicación econométrica del modelo de diferencias en diferencias

\subsection{El modelo de diferencia en diferencia del Programa Empresa Centro:Enfoque econométrico}

Los estudios cuasi experimentales intentan evaluar la efectividad de una intervención comparando dos grupos, uno de los cuales la recibe y el otro no. En ese sentido, en este estudio se aborda y se da respuesta a la interrogante ¿Cuál es la situación de los participantes luego de haberse ejecutado el proyecto en sus diferentes acciones de formación?

Primero, es importante precisar que el presente estudio tiene cobertura parcial y no total para El

\footnotetext{
4 Billorou, Nina, y otros. Guía parta la evaluación de impacto de la formación. OIT, CINTERFOR, p.95.
} 
Tabla n. ${ }^{\circ} 2$

El Salvador. Resultados de la aplicación del método de la diferencia en diferencia sobre los ingresos en el PEC

\begin{tabular}{|l|c|c|c|}
\hline & Después & Antes & \\
\hline Grupo de tratamiento & B & A & B-A \\
\hline Grupo de comparación & D & C & D-C \\
\hline Diferencia & B-D & A-C & DD-A)-(D-C) \\
\hline \multicolumn{3}{|c|}{ Antes } & 176.7 \\
\hline & Después & 180.5 & 16.3 \\
\hline Grupo de tratamiento & 357.2 & 364.6 & $\mathbf{1 6 0 . 4}$ \\
\hline Grupo de comparación & 380.9 & -184.1 & \\
\hline Diferencia & -23.7 &
\end{tabular}

Fuente: Elaboración propia.

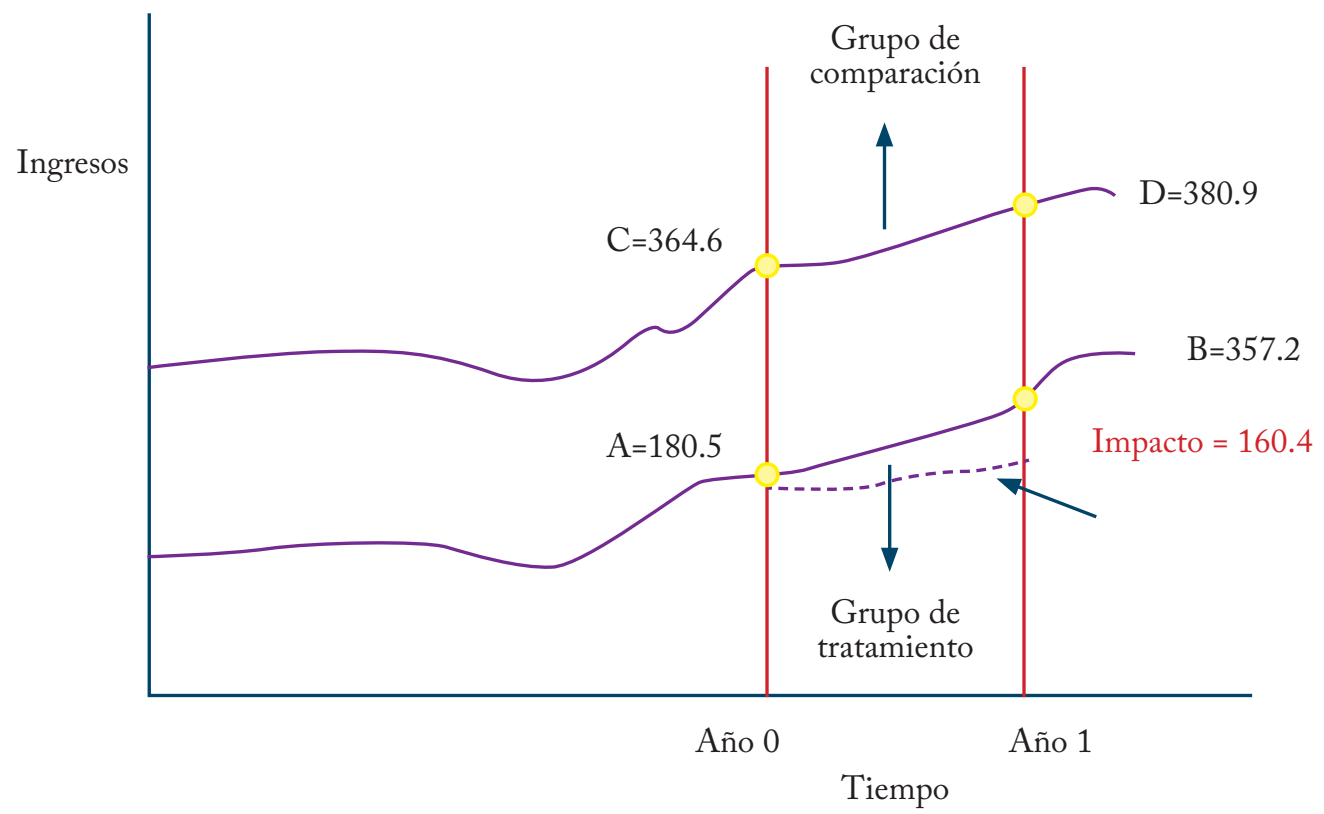

Figura n. ${ }^{\circ}$ 2. El Salvador. Evaluación de impacto del ingreso del PEC. Fuente: elaboración propia. 
Salvador; los beneficiarios del proyecto o "grupo de tratamiento" se comparan con un grupo no beneficiario con similares características. Si este último grupo se selecciona de manera aleatoria, se le denomina "grupo de control"; y si es seleccionado de otra manera, se le llama "grupo de comparación".

La selección del "grupo de tratamiento" no es aleatoria, por lo que no se asume un tipo de diseño experimental sino cuasi experimental. ${ }^{5}$ Además, los costos de este tipo de estudios, cuando la cobertura es completa; es decir, cuando se tiene un alcance nacional, la comparación es compleja y costosa, dado que debe recurrirse a la construcción de modelos computables de equilibrio general, comparación entre países, etc.

Sin embargo, en esta investigación se toma en cuenta, para su evaluación respectiva, al grupo de tratamiento para observar su comportamiento, tomando de referencia al que corresponde al grupo de no participantes. ${ }^{6}$ Para el caso del grupo de participantes para el cual se levantó la información contemplada en la boleta de trabajo, y que se entrenaron en los diferentes cursos de capacitación, jóvenes que no fueron definidos de manera aleatoria, y por consiguiente, las características consideradas de esa manera, en este tipo de análisis, poseen un carácter cuasi experimental.

En términos de procedimiento, se ha seleccionado un grupo de comparación a partir de la

5 Ibidem, p. 98.

6 Si la selección es aleatoria se estaría frente a un diseño experimental. Esto implica que desde el principio se deberá partir de un conjunto bien definido de personas para hacer la selección, tanto para el grupo donde el proyecto realiza la intervención (grupo en tratamiento), como el de control, lo cual no es el caso de la presente consultoría. participación relativa del levantamiento realizado en los departamentos de todo el país. El grupo de comparación se realizó tomando en cuenta la participación relativa del levantamiento por cada carrera de formación profesional. Estos datos muestrales se extrajeron de la Encuesta de Hogares de Propósitos Múltiples 2010 que levanta la Dirección General de Estadística y Censos, DIGESTYC, tomando en cuenta variables específicas de comparación como son los siguientes: sexo, estado familiar, edad, educación, ingreso, etc., con un nivel de confianza del 95\% de que ambas muestras son estadísticamente significativas y que poseen las características similares en esas variables.

Posteriormente, se ha aplicado la técnica "doble diferencia" o "diferencia en la diferencia”; es decir, midiendo los promedios de las muestras del grupo de tratamiento y de comparación o pareo, con lo cual se han medido los impactos respectivos. En síntesis, de los registros que proporcionó INSAFORP se levantó la muestra teórica de 122 participantes de aquellos que fueron sujetos de apoyo con el Programa Empresa Centro. ${ }^{7}$ Además, con la muestra del grupo de pareamiento se han realizado múltiples pruebas en dirección a poseer la consistencia estadística robusta requerida. En síntesis, este método a aplicar, que tiene una larga historia, es el método más común en la evaluación de impacto, debido a que no necesita construcciones específicas, tal como se ha dicho más arriba.

\subsection{Definición y análisis de las variables}

La variable ocupación representa la condición laboral, es decir, si trabaja o no trabaja; variable

\footnotetext{
7 Ver Baker, j. "Evaluación del impacto de los proyectos de desarrollo en la pobreza, Manual para profesionales". Banco Mundial, Washington, D.C., 2000.
} 
dicotómica, es decir, si el participante es ocupado adopta el valor de uno, si no está ocupado adopta el valor de cero de ambos grupos muestrales. ${ }^{8}$ Se espera una relación positiva entre la variable explicativa ocupación y la variable ingresos, que es la variable dependiente. Se espera que en la medida en que exista condición de trabajo, existirá un mayor ingreso, mejorando de esa manera el bienestar de los individuos. A través de esta relación se mide el impacto de la condición de empleabilidad lograda, posteriormente a los cursos de formación, sobre los ingresos del individuo.

Otra variable fundamental es negocio, y está referida a que el individuo posea un negocio. En este caso se espera un signo positivo para esa variable, que es dicotómica, que toma el valor de uno si el individuo se ocupa y tiene un negocio, y cero si no lo tiene. Esto es así ya que la probabilidad de empleabilidad es mayor cuando se cuenta con un negocio propio y su impacto en los ingresos es positivo.

La variable sexo en el modelo de impacto aplicado es una variable dicotómica que toma el valor uno si el individuo es hombre, y el valor de cero si el participante es mujer. No se puede decir de manera categórica que el coeficiente de esta variable tendrá un signo positivo o negativo porque hasta ahora no existe una teoría bien establecida para el nicho de mercado en estudio sobre su comportamiento, básicamente cuando se analiza la participación de la mujer en los mercados de tipo informal, a diferencia del mercado formal del trabajo.

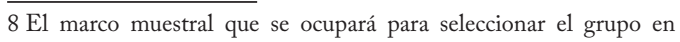
tratamiento y el grupo control, son las personas egresadas del Programa Empresa Centro para los años 2010-2011.
En el modelo, la variable $\mathbf{P}_{\mathrm{i}}$ es una variable binaria que toma el valor de uno si la persona fue participante en los cursos de capacitación, y de cero si el individuo no fue afectado, es decir, no participó en los cursos de capacitación. En este caso, dado que los efectos que se esperan de haber participado en los cursos de formación es el hecho de que se incremente su nivel de bienestar, visto en sus ingresos personales y que exista un impacto positivo por ese hecho. En síntesis, se espera un coeficiente positivo de la variable que captura en el modelo la diferencia entre los afectados por los cursos de capacitación y los que no tuvieron esa oportunidad.

\subsubsection{E1 modelo}

El modelo de regresión que se adopta se describe así:

$$
Y_{i t}=\alpha+\beta P_{t}+X_{t}+\mu_{t}
$$

donde:

- i representa el número de variables.

- t representa para nuestro caso, el número de observaciones, es decir $\mathrm{N}=244$.

- De esa manera $X_{\text {it }}$ es el vector de las variables explicativas.

- $\mathbf{Y}_{\mathrm{it}}$ (ingresos de los individuos) corresponde a la variable dependiente a considerar.

- $\mathbf{P}_{\mathrm{it}}$ es la variable designada para las personas participantes o grupo de tratamiento.

La $\mu_{i t}$ representa el término de la perturbación estocástica. 


\subsubsection{Forma funcional del modelo}

En esta sección se proponen los factores que inciden y determinan los impactos en el bienestar de los individuos; es decir, los ingresos. Por consiguiente, se plantearán las variables que producen esos impactos respectivos. La importancia de este suceso es que esta información desde el punto de vista estadístico se podrá inferir para el marco muestral con el que se ha trabajado y definir las tendencia para el país. Utilizando el paquete SPSS v. 22 se correrá la forma funcional, la cual tendrá la siguiente estructura:

$Y_{i t}=\alpha+\rho_{1}$ Ocupado $_{t}+\rho_{2}$ Negocio $_{t}+\rho_{5}$ Sexo $_{t}+\beta P_{i}+\mu_{t}$

Donde:

- $\mathrm{Y}_{\mathrm{it}}=$ Ingreso de los participantes del grupo de tratamiento y no participantes; es decir, del grupo de comparación.

- Ocupado $_{\text {it }}=$ Individuos que sí poseen un trabajo=1 y si no lo posee $=0$ del grupo de tratamiento y del grupo de comparación.

- Negocio $_{\text {it }}=$ Individuos que cuentan con un negocio $=1$, y si no=0, del grupo de tratamiento y del grupo de comparación.

- Sexo $_{\text {it }}=$ Los individuos que sean hombres=1,y si son mujeres $=0$, del grupo de tratamiento $y$ del grupo de comparación.

- $\mathbf{P}_{\mathrm{it}}=$ Es la variable que indica que si el individuo recibió cursos de capacitación $=1, \mathrm{y}$ si no $=0$.

En resumen, se espera que los coeficientes, es decir todos los parámetros, tengan un signo positivo, a excepción del que corresponde a sexo, que podría adoptar positivo con algunas reservas.

\subsection{Hipótesis general del modelo}

El bienestar de los participantes en el Programa Empresa Centro, medido a través de los ingresos de los jóvenes, es determinado y explicado por la formación o entrenamiento recibido a través de las carreras de formación profesional, realizadas durante los años 2010 y 2011 por INSAFORP y de otras variables descritas más arriba.

\subsection{Supuesto específico: la homocedasticidad}

El modelo clásico de regresión lineal para estimaciones de corte transversal por lo general no está afectado por la autocorrelación de cada una de las perturbaciones, es decir que $E\left(\mu_{i} \mu_{i}\right)=0$ donde $\mathrm{i} \neq \mathrm{j}$. Aunque la correlación generalmente predomina en series de tiempo.

En el modelo que se ha considerado para medir el nivel de bienestar y los impactos de la empleabilidad alcanzada y otras variables, se basa en el supuesto de que la varianza de cada término de perturbación $\boldsymbol{\mu}_{i t}$, es condicional a los valores escogidos de las variables explicativas, es un número constante igual a $\boldsymbol{\sigma}^{2}$. Este es el supuesto de homocedasticidad o igual (homo) dispersión (cedasticidad), es decir, igual varianza. En símbolos $E\left(\sigma_{i}^{2}\right)=\sigma^{2} i=1,2, \ldots, N$

\section{Interpretación de resultados}

\subsection{Aplicación del Modelo e Interpretación de Resultados}

Los resultados del modelo muestran una relación positiva entre el nivel de ocupación con impacto sobre la variable de ingresos con una relación positiva como lo esperado 
y es altamente significativa. Eso se explica que en la medida en que se tenga y se acceda a un empleo, se tiene una probabilidad en términos promedio de incrementar el ingreso en 69.7 puntos de diferencia sobre aquellos que no gozan de esa oportunidad de formación profesional. Esto es el impacto del PEC, que permite y asegura reducir los niveles de pobreza de los participantes del programa.

La variable negocio, con 79.2 puntos por encima de los que no participaron de los cursos de formación, e indica que el signo es el esperado, es decir, positivo; además que el resultado es estadísticamente significativo. Se sugiere considerar esta variable en el modelo.

La variable sexo se comporta con fuerte impacto, pues además de que el signo es el esperado, y es altamente significativa con un resultado de $\mathbf{5 6 . 7}$ puntos de diferencia con respecto al sexo femenino, de aquellos no participantes en los cursos de formación, y que indica que efectivamente esta variable impacta cuando es hombre, frente al caso de ser mujer en los cursos de formación.

La variable $\mathbf{P}$ que recibió el individuo que cursó carreras de formación profesional tiene un impacto positivo sobre los ingresos promedios mensuales y se encuentra con el signo esperado. Es, además, estadísticamente significativo el resultado. Esto quiere decir que su coeficiente 150.4 es la diferencia de los participantes con respecto a los que no participaron en los cursos de formación impartidos por INSAFORP.

\section{Variable dependiente Ingresos en USD promedios mensuales.}

\section{Método de Mínimos Cuadrados Ordinarios.}

Muestra $=244$ participantes de grupo de tratamiento y grupo de comparación.

Programa SPSS v.22.

Tabla n. 3

El Salvador. Aplicación de modelo econométrico del grupo de tratamiento del PEC y grupo de comparación de la EHPM

\begin{tabular}{|l|c|c|c|c|c|}
\hline & \multicolumn{2}{|c|}{ Coeficientes no estandarizados } & $\begin{array}{c}\text { Coeficientes } \\
\text { estandarizados }\end{array}$ & & \\
\hline \multicolumn{1}{|c|}{ Modelo } & $\mathrm{B}$ & Error estándar & Beta & $\mathrm{t}$ & Sig \\
\hline 1 (Constante) & 104.170 & 22.052 & & 4.724 & .000 \\
\hline PARTICIPANTES & 150.375 & 24.471 & .363 & 6.145 & .000 \\
\hline OCUPADOS & 69.740 & 24.834 & .167 & 2.808 & .005 \\
\hline SEXO & 56.674 & 24.046 & .136 & 2.357 & .019 \\
\hline NEGOCIO PROPIO & 79.175 & 40.970 & .112 & 1.933 & .054 \\
\hline
\end{tabular}

a. Variable dependiente: INGRESOS

Fuente: Elaboración propia. 


\section{Conclusiones}

El Programa Empresa Centro 2010-2011 tiene un fuerte impacto en la empleabilidad y en los ingresos de los participantes. La oferta recibida a través de los centros de formación profesional acreditados por INSAFORP tiene un fuerte impacto en las condiciones básicas para la empleabilidad de los participantes. El resultado de la diferencia entre el grupo de tratamiento y el grupo de comparación en relación al empleo, indica que la velocidad de incremento del empleo en el grupo de tratamiento es superior en 437 puntos versus el grupo de comparación. Los participantes del PEC incrementaron su inserción productiva en 195.4\%; mientras que el grupo de comparación lo hizo con apenas un 14.5\%.

En relación con los ingresos, el resultado de la diferencia entre el grupo de tratamiento y grupo de comparación indica que la velocidad de incremento de los ingresos en el grupo de tratamiento es superior en 160.4 puntos versus el grupo de comparación.

La variación de los ingresos del grupo de tratamiento es de $97.9 \%$ mientras que la variación del grupo de comparación es de 4.5\%; esto indica que los cursos de formación tuvieron un impacto positivo en los ingresos de los participantes.

- Los impactos cuantitativos y cualitativos de los cursosimpartidos generanunincremento del bienestar en los participantes. Los resultados de la evaluación de impacto de la oferta de las carreras de formación generan empleabilidad y mejoran los ingresos en el marco del Programa Empresa Centro; $y$ en consecuencia, en el incremento de las condiciones de vida de la población participante, impactos positivos, logrando los objetivos del Programa frente aquellos que no lograron participar en los años 2010 y 2011.

\section{- E1 Programa Empresa Centro 2010-2011} posee trazabilidad de género. De nuevo se registra un dato sumamente importante en términos de los participantes. Las mujeres experimentan también oportunidades, al igual que los hombres, con propensión a la empleabilidad, dado que las especialidades fueron aceptadas y solicitadas por parte de cada uno de los centros de formación.

\section{- En el territorio, las oportunidades por impulsar} la empleabilidad no se han utilizado en todo su dinamismo y entusiasmo. En el proceso de búsqueda de empleo, las instituciones que mayormente fueron citadas como las que han ofrecido orientación han sido los centros de formación profesional y cada una de las empresas asociadas con el Programa.

- La educación de base de los participantes requiere de más formación formal para elevar los niveles de productividad. Los jóvenes participantes manifestaron que las razones que obstaculizan la contratación en el sector formal; en primer lugar, es el hecho de que el empleador requiere de personal experimentado; en segundo lugar, los participantes consideran que muy poco pagan los empleadores; y en tercer lugar, manifestaron que el nivel de escolaridad era insuficiente para ser contratado. ${ }^{9}$

\footnotetext{
9 PNUD, Informe de Desarrollo Humano El Salvador 2013, Imaginar un nuevo país. Hacerlo posible, Resumen, p.25.
} 
- Persisten limitantes para la adecuada aplicabilidad de los conocimientos adquiridos por los participantes. En la medición del impacto sobre los cursos recibidos sobre su aplicabilidad o no en el empleo, los participantes tienen la opinión de que ha sido de mucha utilidad tanto para el trabajo como para poner un negocio.

- La metodología utilizada para comparar los resultados entre el grupo de tratamiento y el de comparación posee un rigor analítico que genera confianza en la evaluación del Programa. La evaluación de impacto del programa, comparando los resultados alcanzados por los egresados, respecto al grupo de comparación, muestra un impacto positivo en todas las variables evaluadas.

\section{Bibliografía}

Abdala, E. (2004). Formación y empleabilidad de jóvenes en América Latina. En Molpeceres Pastor, M. (coord.), Identidades y formación para el trabajo.Montevideo: Organización Internacional del Trabajo (OIT)/Centro Interamericano de Investigación y Documentación sobre Formación Profesional (CINTERFORP).

Agencia de noticias ACAN-EFE. (2008). La economía crecerá poco. La Prensa Gráfica.

Baker, J. (2000). Evaluación del impacto de los proyectos de desarrollo en la pobreza, Manual para profesionales. Washington, D.C.: Banco Mundial.

BancoInteramericanode Desarrollo.Recuperado de: http://www.iadb.org/NEWS/articledetail. cfm? Language $=$ Sp\&parid $=4 \&$ art Type $=W S \&$ $\operatorname{artid}=4377$
Billorou, N. y otros. (2011). Guía para la Evaluación de Impacto de la Formación. Montevideo: CINTERFOR-OIT.

Brown, A. (1978). Regression et Correlation. Quebec: Press de l'Université Laval.

Damodar, G. (1992). Econometría, Segunda edición. Editorial: McGraw.

Dirección General de Estadísticas y Censos (DIGESTYC). (Varios años). Encuesta de Hogares de Propósitos Múltiples. San Salvador: DIGESTYC. Recuperado de: http://www. digestyc.gob.sv/

División de Estadísticas de la Organización de Naciones Unidas. (2008). Indicadores de los Objetivos de Desarrollo del Milenio. Recuperado de: http://unstats.un.org/unsd/mdg/Host. aspx?Content=Indicators/OfficialList.htm

Facultad Latinoamericana de Ciencias Sociales (FLACSO). (2005). Mapa de Pobreza: Politica Social y Focalización. San Salvador: Autor.

Gallart, M. (2003). La formación para el trabajo y los jóvenes en América Latina. Santiago de Chile: CEPAL.

Glejberman, D. (2007). Curso de muestreo aplicado a Encuestas Económicas, DIGESTYC.

IHS Global (s.f.). EViews User's Guide, version 3.0. California: Autor.

Instituto Salvadoreño de Formación Profesional (INSAFORP). (2013). Evaluación de impacto: incidencia de la capacitación para el trabajo en la 
incorporación al empleo, de la población egresada del programa habilitación para el trabajo, años 2001 y 2002. San Salvador: Autor.

Johnston, J. (1985). Methodes Econometriques, tome 1 et 2 3a. Edition. París: Económica.

Ley de Formación Profesional. Diario Oficial de E1 Salvador. San Salvador, E1 Salvador, 29 de julio de 1993.

Márquez, G., Chong. A., Duryea, S., Mazza, J., Nopo, H. (2008). ¿Los de afuera? Patrones cambiantes de exclusión en América Latina y el Caribe.Washington,D.C.: Banco Interamericano de Desarrollo.

Ministerio de Educación (2005). Plan Nacional 2021, Metas y Politica para Construir el País que Queremos. Primera edición. San Salvador: MINED.

Núñez, S. (2002). Evaluación de impacto de un programa de entrenamiento laboral a través de modelos econométricos. El Caso de Proyecto Joven. República de Argentina. (Monografía de Graduación) Georgetown University.

Organización de Estados Americanos. Recuperado de: http://www.sedi.oas.org/ddse/ espanol/cpo_trab_rial_jovenes.asp\#_Hlk3
Organización Internacional del Trabajo (OIT). (2013). La OIT en América Latina y el Caribe, Avances y perspectivas. Lima: Autor.

Organización Internacional del Trabajo (OIT). (2013). Tendencias mundiales del desempleo. Lima: Autor.

Organización Internacional del Trabajo (OIT). Informe mundial sobre salarios 2012/2013, Los salarios y el crecimiento equitativo. Ginebra: Autor.

Organización Internacional del Trabajo (OIT). La crisis del empleo juvenil: Un llamado de acción, Resolución y conclusiones de la 101a. Reunión de la Conferencia Internacional del Trabajo, 2012. Ginebra.

Programa de Naciones Unidas para el Desarrollo (PNUD). Informe de Desarrollo Humano El Salvador 2013, Imaginar un nuevo país. Hacerlo posible. San Salvador: Autor.

Ranieri, R.; Almeida R. (s.f.). What is Inclusive Growth? International Policy Centre for Inclusive Growth (IPC-IG).

Seminario rial sobre empleo de jóvenes, llevado a cabo en Río de Janeiro, mayo 20 y 21 del año 2008.

Weller, J. (2007). La Inserción laboral de los Jóvenes, Características tensiones y desafíos, Revista de la CEPAL, n. .92. 